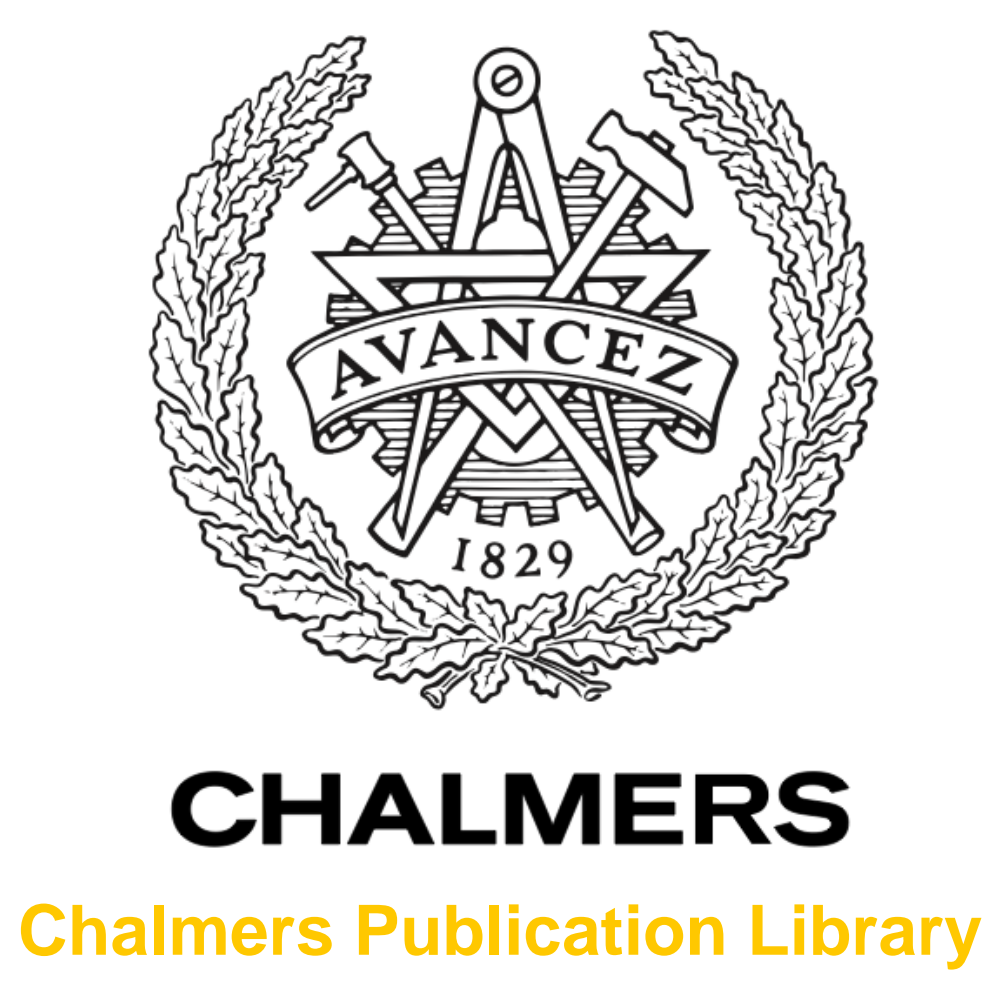

\title{
Bandgap engineering in nonlinear silicon nitride waveguides
}

This document has been downloaded from Chalmers Publication Library (CPL). It is the author's version of a work that was accepted for publication in:

\section{Optical Fiber Communication Conference (OFC)}

Citation for the published paper:

Krückel, C. ; Fülöp, A. ; Andrekson, P. et al. (2017) "Bandgap engineering in nonlinear silicon nitride waveguides". Optical Fiber Communication Conference (OFC)

Downloaded from: http://publications.lib.chalmers.se/publication/248867

Notice: Changes introduced as a result of publishing processes such as copy-editing and formatting may not be reflected in this document. For a definitive version of this work, please refer to the published source. Please note that access to the published version might require a subscription. 


\title{
Bandgap engineering in nonlinear silicon nitride waveguides
}

\author{
Clemens J. Krückel, Attila Fülöp, Peter A. Andrekson and Víctor Torres-Company \\ Photonics Laboratory, Department of Microtechnology and Nanoscience, Chalmers University of Technology, SE-412 96 Gothenburg, Sweden \\ kruckel@chalmers.se
}

\begin{abstract}
We show that controlling the bandgap of $\mathrm{SiN}$ provides an additional degree of freedom for engineering waveguides for nonlinear optics. We show an optimized structure with $\gamma \cdot \max L_{\text {eff }}=$ $0.17 \mathrm{rad} / \mathrm{W}$ and absence of nonlinear loss.

OCIS codes: (130.4310) Integrated optics: Nonlinear; (160.6000) Materials: Semiconductor materials; (220.4241) Optical design and fabrication: Nanostructure fabrication.
\end{abstract}

\section{Introduction}

Silicon rich nitride is a CMOS-compatible and robust material platform suitable for integrated linear [1] and nonlinear optics [2,3]. The increased silicon content compared to stoichiometric silicon nitride $\mathrm{Si}_{3} \mathrm{~N}_{4}$ provides low material stress and higher nonlinearities. We have recently demonstrated octave-spanning supercontinuum generation using low energy pulses directly obtained from a femtosecond fiber oscillator [4]. These results indicate that the material is not limited by multiphoton absorption and it covers a broad transparency window.

However, the detailed impact of the silicon content on the optical properties has not been analyzed in detail yet. In this contribution we evaluate the linear and nonlinear properties when changing the relative composition of silicon and nitride. This study reveals that there is an optimum composition with a bandgap above the two-photon absorption limit in the telecom window. Rectangular waveguides are fabricated with calculated anomalous dispersion in a highly reproducible fabrication process. The corresponding relative nonlinear phase shift is $0.17 \mathrm{rad} / \mathrm{W}$. To put things in context, this relative nonlinear phase shift is around $30 \%$ larger than in $\mathrm{Si}_{3} \mathrm{~N}_{4}$ waveguides [5]. This structure opens up new opportunities for realizing nonlinear optical processing of telecom signals.

\section{Fabrication and material properties}

We followed a similar fabrication process as the one described in [2]. The geometry consists of a silicon nitride core embedded in silicon dioxide. The deposition of silicon nitride was done in an LPCVD furnace (Centrotherm) where silicon and nitrogen were supplied via the precursor gases dichlorsilane (DCS) and ammonia $\left(\mathrm{NH}_{3}\right)$. In order to change the atomic composition of the $\mathrm{Si}_{\mathrm{x}} \mathrm{N}_{\mathrm{y}}$ film, the gas flow ratio of DCS and $\mathrm{NH}_{3}$ is modified. An increase in DCS: $\mathrm{NH}_{3}$ flow ratio yields in an increase in silicon content of the deposited silicon nitride film [2].

Four different $\mathrm{Si}_{\mathrm{x}} \mathrm{N}_{\mathrm{y}}$ compositions were fabricated under similar conditions, starting from a DCS: $\mathrm{NH}_{3}$ ratio of 4 up to the maximum possible ratio of 16.67 given by the furnace. A thin film of stoichiometric silicon nitride $\mathrm{Si}_{3} \mathrm{~N}_{4}$ (DCS:NH3 ratio of 0.27) was also fabricated and shown in the following figures for comparison. It should be noted that a similar study has been carried out in a PECVD furnace [6] with different deposition features.

The silicon rich nitride films ( $\sim 700 \mathrm{~nm}$ thickness) were patterned with optical lithography followed by $\mathrm{CHF}_{3}$ based dry etching to form a waveguide core of $\sim 1.65 \mu \mathrm{m}$ in width. It is worth noting that the presented silicon rich nitride films benefit from the increased silicon content in terms of low-stress properties. The thickness of $700 \mathrm{~nm}$ could be deposited in a single step without cracks. The thickness ensures that all fabricated waveguides operate in the anomalous dispersion regime in the $1.5 \mu \mathrm{m}$ band.

An ellipsometer analysis was carried out to investigate thickness, refractive index and optical bandgap of the different silicon nitride films. In order to represent the ellipsometer data of the film, we used the Tauc-Lorentz oscillator model [7]. The model matches the film characteristics by fitting a dielectric function to the measurement data with the optical bandgap as one of the fitting parameters. The available ellipsometer covers the wavelength range from 245 $\mathrm{nm}$ to $1000 \mathrm{~nm}$. The measured refractive index within this spectral range is shown in Fig. 2.a. Above one micron, the refractive index function is extrapolated using the fitting parameters as shown in the shadowed area in Fig. 2.a. It becomes clear when comparing the compositions that an increase in gas flow ratio results in an increase in refractive index. At $1550 \mathrm{~nm}$ wavelength the gradual increase of refractive index with DCS: $\mathrm{NH}_{3}$ gas flow ratio is illustrated in Fig. 2.b. The bandgap dependence on the material composition is shown in Fig. 2.c. Starting above $3 \mathrm{eV}$ for $\mathrm{Si}_{3} \mathrm{~N}_{4}$ the bandgap quickly decreases for a higher DCS: $\mathrm{NH}_{3}$ ratio. All materials show a bandgap above the two-photon absorption (TPA) limit of around $1.6 \mathrm{eV}$ for wavelength at $1550 \mathrm{~nm}$, thus no nonlinear loss is expected in the telecommunications 


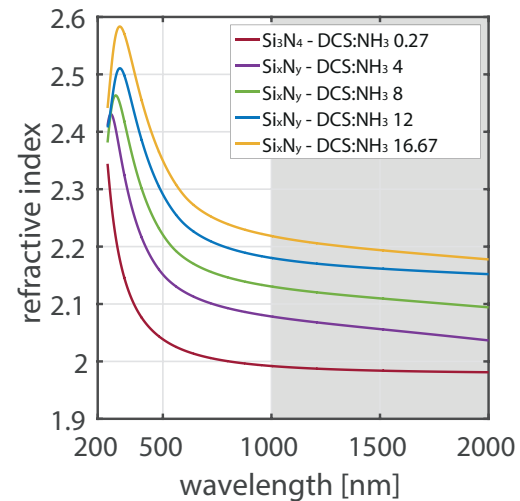

(a)

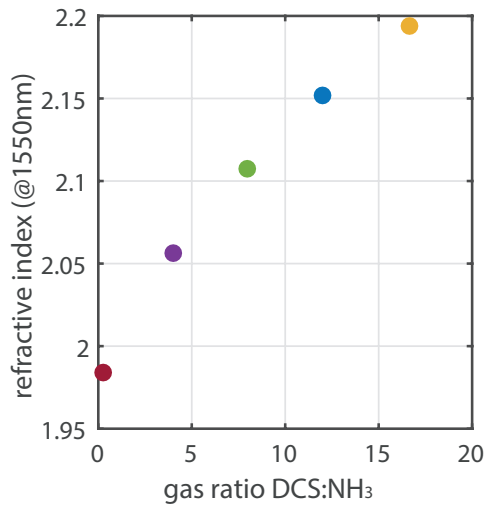

(b)

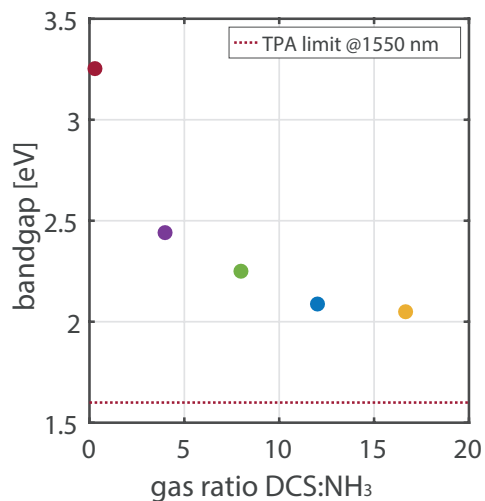

(c)

Fig. 1. a) Measured refractive index as a function of wavelength for different $\mathrm{Si}_{3} \mathrm{~N}_{4}$ compositions. The shadowed area shows extrapolated data from the measurements. b) Refractive index at $1550 \mathrm{~nm}$ as a function of DCS: $\mathrm{NH}_{3}$ ratio. c) Optical bandgap as a function of DCS: $\mathrm{NH}_{3}$ ratio.

bands (see Sect.4).

\section{Waveguide propagation losses}

The impact of the silicon rich nitride composition on the waveguide loss has been measured using the cut-back method with three different lengths $(1,3$ and $4 \mathrm{~cm})$. Each waveguide was characterized in a spectrally resolved manner for wavelengths from 1510 to $1610 \mathrm{~nm}$. For each characterization the polarization was tuned for maximum throughput and the coupling optimized at $1550 \mathrm{~nm}$. For all compositions and wavelengths the coupling loss was around $4 \mathrm{~dB}$ per facet. In order to show the repeatability of the waveguide characteristics on the wafer, five independent waveguides were characterized per composition and Fig. 2 shows the resulting mean and standard deviation of the propagation loss for each of the fabricated compositions. It becomes clear that the propagation loss decreases with gas flow ratio, although the DCS: $\mathrm{NH}_{3}$ ratios of 8 and 4 show almost similar performance with waveguide losses around $1.4 \mathrm{~dB} / \mathrm{cm}$ at $1550 \mathrm{~nm}$. In the context of nonlinear experiments this would lead to a maximum effective length (max $L_{\text {eff }}$ ) of around $3.1 \mathrm{~cm}$. The maximum effective length indicates the optimum waveguides length for nonlinear interaction and is used later in the calculations. Increased absorption at shorter wavelength with a peak around $1520 \mathrm{~nm}$ (likely due to hydrogen absorption) is visible for all compositions.

\section{Nonlinear properties}

The nonlinear properties of waveguides with different silicon rich nitride compositions were evaluated in a dual-pump experiment [8]. For this, the nonlinear phase shift $\left(\phi_{\mathrm{nl}}=\gamma \cdot P_{\text {input }} \cdot L_{\mathrm{eff}}\right)$ was measured in $1 \mathrm{~cm}$ long waveguides and by
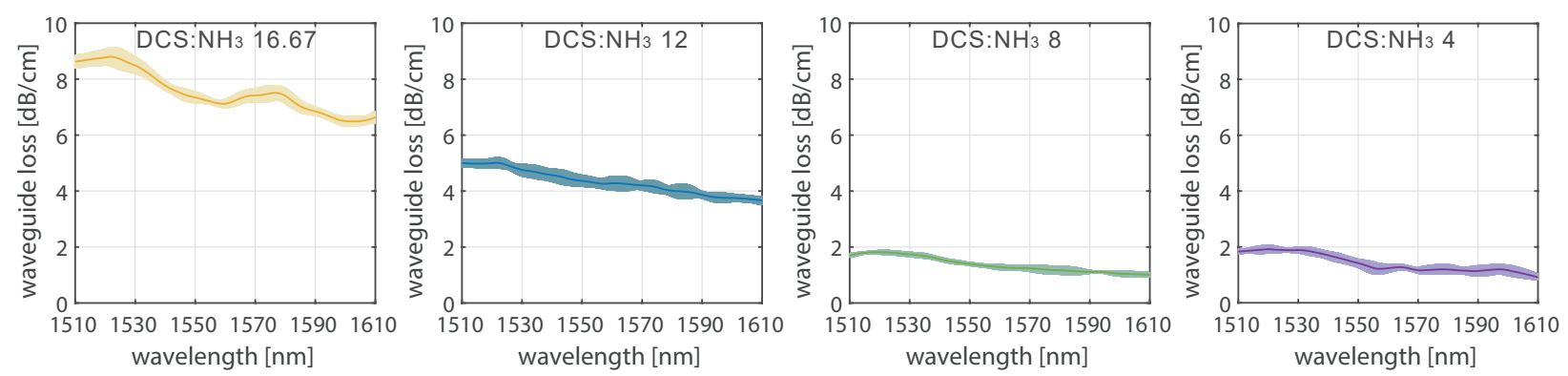

Fig. 2. Waveguide loss as a function of wavelength for silicon rich nitride waveguides fabricated with different DCS: $\mathrm{NH}_{3}$ gas flow ratios. 


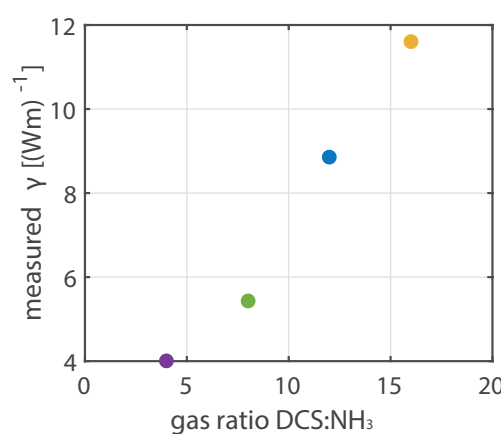

(a)

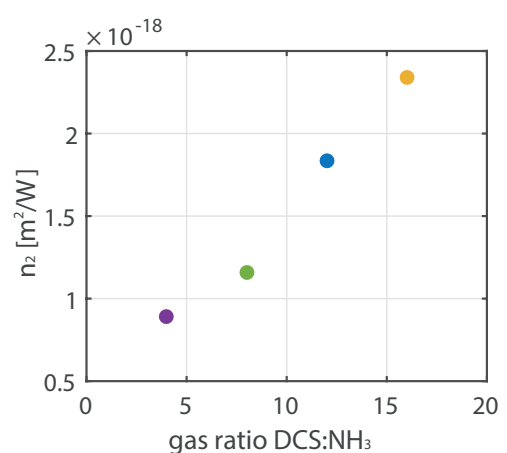

(b)

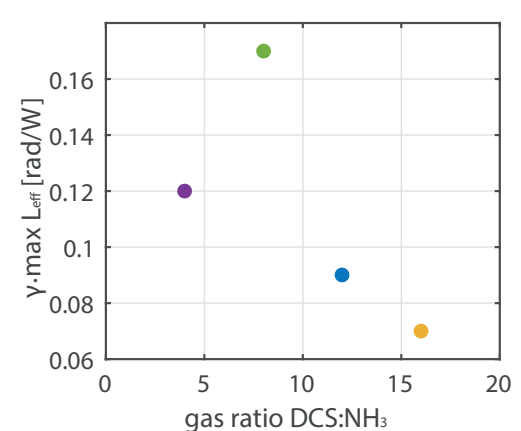

(c)

Fig. 3. a) Measured nonlinear parameter $\gamma$ as a function of DCS:NH $\mathrm{NH}_{3}$ ratio. b) Estimated nonlinear Kerr coefficient as a function of optical bandgap. c) Maximum relative nonlinear phase shift $\gamma$. $\max L_{\text {eff }}$ as a function of DCS: $\mathrm{NH}_{3}$ ratio.

knowing the input power $P_{\text {input }}$ and effective length $L_{\text {eff }}$ the nonlinear parameter $\gamma$ was calculated [2]. No nonlinear loss was observed for the coupled powers (several hundreds of $\mathrm{mW}$ ) for the different compositions, as expected from the bandgap measurements. A summary of the measurements is presented in Fig. 3.a where $\gamma$ is shown for waveguides with different silicon nitride compositions. The plot reveals an increase in nonlinear parameter with gas flow ratio where the maximum achieved $\gamma$ is $11.6(\mathrm{~W} \cdot \mathrm{m})^{-1}$. This is about one order of magnitude higher than in $\mathrm{Si}_{3} \mathrm{~N}_{4}$ waveguides of comparable dimensions [5]. However, as the nonlinear parameter $\gamma=\frac{2 \pi n_{2}}{\lambda A_{\mathrm{eff}}}$ includes waveguide confinement via the effective area (lower $A_{\text {eff }}$ for higher silicon content), for a fundamental comparison on material level the nonlinear Kerr coefficient $n_{2}$ has to be compared. From the evaluated $\gamma$ of the presented measurement we calculate the $n_{2}$ using mode solver based simulations for $A_{\text {eff }}$ of the fundamental TE-mode. The results are presented in Fig. 3.b. A maximum Kerr coefficient of around $2.4 \cdot 10^{-18} \mathrm{~m}^{2} / \mathrm{W}$ has been achieved for the material with the largest silicon content which is an order of magnitude larger than that of $\mathrm{Si}_{3} \mathrm{~N}_{4}$.

In order to characterize the impact of both the waveguide nonlinearities and losses, we calculate the maximum relative nonlinear phase shift $\gamma \cdot \max L_{\text {eff }}$ for the different compositions. Figure Fig. 3.c shows the results and indicates an optimum composition for maximum nonlinear phase shift.

\section{Conclusion}

We have presented silicon nitride films with different compositions achieved by gas flow tuning during LPCVD deposition. This allows selective optical bandgap engineering which enables the control of linear and nonlinear material properties. We have demonstrated that an increased silicon content brings the advantage of crack free fabrication of thick waveguides and increased values of refractive index and nonlinearities. The nonlinearities and loss characteristics lead to a maximum $\gamma \cdot \max L_{\text {eff }}$ product of $0.17 \mathrm{rad} / \mathrm{W}$ corresponding to composition $\mathrm{DCS}: \mathrm{NH}_{3}$ 8 in Figs. 2 and 3. In summary, this material composition brings advantages in fabrication and combines optimum nonlinearities and losses with high power handling capabilities, suitable to implement efficient systems for nonlinear integrated optics.

\section{References}

[1] Philipp et al.,'Amorphous silicon rich nitride optical waveguides for high density integrated ...”, Electr. Lett.,40,419-421 (2004)

[2] Krückel et al.,'Linear and nonlinear characterization of low-stress high-confinement ...", Opt. Expr.,23,25827-25837 (2015)

[3] Wang et al.,"'Supercontinuum generation in bandgap engineered back-end CMOS ...", Laser and Phot. Rev.,9,498 (2015)

[4] Liu et al.,"Octave-spanning supercontinuum generation in a silicon-rich nitride waveguide”, Opt. Lett.,41,2719-2722 (2016)

[5] Moss et al.,"New CMOS-compatible platforms based on silicon nitride and Hydex for ...", Nature Photon.,7,597-607 (2013)

[6] Lacava et al.,"CMOS-compatible Silicon-Rich Nitride Waveguides for ...", in Proceedings of CLEO(2016), paper STu4Q.7

[7] Jellison et al.,"Parametrization of the optical functions of amorphous materials in the ...", Appl. Phys. Lett.,69, 371 (1996)

[8] Boskovic et al.,'Direct continuous-wave measurement of $\mathrm{n}_{2}$ in various types of ...”, Opt. Lett.,21,1966-1968 (1996)

This work is supported by the European Research Council under grant agreement ERC-2011-AdG-291618 PSOPA, the Swedish Research Council and the Knut och Alice Wallenberg Foundation. 